\title{
Accuracy of the neurosurgeons estimation of extent of resection in glioblastoma
}

\author{
Sümeyye Sezer ${ }^{1} \cdot$ Martin J. van Amerongen ${ }^{2} \cdot$ Hans H. K. Delye $^{1} \cdot$ Mark ter Laan $^{1}$
}

Received: 16 April 2019 / Accepted: 24 September 2019 / Published online: 28 October 2019

(C) The Author(s) 2019

\begin{abstract}
Background The surgeons' estimate of the extent of resection (EOR) shows little accuracy in previous literature. Considering the developments in surgical techniques of glioblastoma (GBM) treatment, we hypothesize an improvement in this estimation. This study aims to compare the EOR estimated by the neurosurgeon with the EOR determined using volumetric analysis on the postoperative MR scan.

Methods Pre- and post-operative tumor volumes were calculated through semi-automatic volumetric assessment by three observers. Interobserver agreement was measured using intraclass correlation coefficient (ICC). A univariate general linear model was used to study the factors influencing the accuracy of estimation of resection percentage.

Results ICC was high for all three measurements: pre-operative tumor volume was 0.980 (0.969-0.987), post-operative tumor volume 0.974 (0.961-0.984), and EOR 0.947 (0.917-0.967). Estimation of EOR by the surgeon showed moderate accuracy and agreement. Multivariable analysis showed a statistically significant effect of operating neurosurgeon $(p=0.01)$, use of fluorescence $(p<0.001)$, and resection percentage $(p<0.001)$ on the accuracy of the EOR estimation.

Conclusion All measurements through semi-automatic volumetric analysis show a high interobserver agreement, suggesting this to be a reliable assessment of EOR. We found a moderate reliability of the surgeons' estimate of EOR. Therefore, (early) postoperative MRI scanning for evaluation of EOR remains paramount.
\end{abstract}

Keywords Extent of resection · Glioblastoma multiforme $\cdot$ Interobserver agreement $\cdot$ Tumor volume measurement

\section{Introduction}

Glioblastoma (GBM) represents $47.1 \%$ of malignant primary brain tumors, making it the most common type of malignant primary brain tumor [1]. Even though survival rates have improved, prognosis of GBM remains poor. Surgery aimed at as much resection as safely possible is the main treatment option.

This article is part of the Topical Collection on Tumor - Glioma

Electronic supplementary material The online version of this article (https://doi.org/10.1007/s00701-019-04089-8) contains supplementary material, which is available to authorized users.

Mark ter Laan

Mark.terLaan@radboudumc.nl

1 Department of Neurosurgery, Radboud University Medical Center, Geert Grooteplein Zuid 10, 6525 GA Nijmegen, The Netherlands

2 Department of Radiology, Radboud University Medical Center, Geert Grooteplein Zuid 10, 6525 GA Nijmegen, The Netherlands
Increasing the extent of resection (EOR) of GBM is associated with prolonged survival [2]. Also, adjuvant radiochemotherapy showed higher survival rates in patients with complete resection $(\mathrm{EOR} \geq 90 \%)$, compared with partial resection $(\mathrm{EOR}<90 \%)$ [3].

Using volumetric analysis, it is possible to quantify the EOR using MR imaging. Prior studies using semi-automated methods for this purpose found a high interobserver agreement $[4,5]$. However, when manual segmentation is used, a low interobserver agreement in the assessment of tumor resection rates on magnetic resonance imaging (MRI) is described. This applies particularly for post-operative tumor volume and residual tumor volume [6].

Before the general use of post-operative scanning, intraoperative estimation by the neurosurgeon was used to determine partial, subtotal, or total tumor resection. The only study that compared this estimation with the presence of residual tumor mass on a MR image, dates back to 1994 [7]. It showed that neurosurgeons often underestimate the presence of residual tumor. Residual tumor mass was three times more often 
seen on early post-operative MRI than estimated by the neurosurgeon [7].

With the advancement of microsurgical techniques and the introduction of adjuncts such as fluorescence, we hypothesize a high accuracy of the surgeons' estimation of the EOR. Possibly, the post-op MRI scan can be omitted after resection using fluorescence. This study aims to compare the EOR estimated by the neurosurgeon with the EOR determined using volumetric analysis on post-operative MR images.

\section{Methods}

\section{Participants}

Because the study implied no burden for the patients, ethical approval was waived by the ethical committee. In this retrospective study, patient characteristic, operation details, and tumor characteristics were prospectively collected. Therefore, no informed consent was required. Adult patients ( $\geq 18$ years old) who underwent a resection for GBM were included. Exclusion criteria were as follows: (1.) patients without a pre-operative contrast-enhanced MR image (i.e., less than $48 \mathrm{~h}$ before surgery) or without an early postoperative MRI (i.e., within $72 \mathrm{~h}$ after surgery) and (2.) patients that had prior treatment for the target tumor.

\section{Test methods}

All patients were treated according to protocol; neuronavigation was used in all patients and 5-ALA was used for intra-operative fluorescence in 22 patients. The EOR by the neurosurgeon was estimated by the operating surgeon post-operatively $\left(E_{\text {Surgeon }}\right)$, before the post-operative MR scan was made. This estimation was based on intra-operative microscopic visualization and neuronavigation. Post-operative MR imaging was performed within $72 \mathrm{~h}$ after surgery.

Pre- and post-operative T1-weighed MR images (slice thickness $1.0 \mathrm{~mm}$ ) with and without gadolinium contrast were used for semi-automated segmentation of the tumor. Tumor volumes were calculated through semi-automated volumetric analysis using Brainlab software (Brainlab, iPlanNet version 2.3.1.215.1, Munich, Germany) by three observers (a neurosurgeon, a radiologist, and a trained medical student). Previous literature stated the use of this method to be reliable for tumor measurements $[4,5]$. All observers are skilled in interpreting MR images for brain tumors. Each observer was blinded for the data measured by the other observers and for the surgeons' estimate. The neurosurgeon did not measure the tumors of his own patients. For this reason, there was a second neurosurgeon measuring these patients' MR images.

Our definition of tumor volume was in accordance with previous descriptions [6]: the contrast enhancing mass, including the possibly present necrotic area. All three observers were instructed to include any tissue with a high post-contrast signal in their estimation. The minimal detectable residual would be one voxel, which corresponds with $0.954 \mathrm{~mm}^{3}$ using our settings. To eliminate hemorrhage inclusion in post-operative tumor volume (Post-opTV) determination, any parts around the resection area with high signal on T1-weighed image without contrast were subtracted from the hyperintense mass on the contrast-enhanced T1-weighed image. Extent of resection (EOR) was calculated as:

$$
\begin{aligned}
\text { EOR }(\text { in } \%)= & ((\text { Pre }- \text { opTV }- \text { Post }- \text { opTV }) / \text { Pre }- \text { opTV }) \\
& \times 100 \%
\end{aligned}
$$

This EOR calculation was done for the measurements of the surgeon, the radiologist, and the student, respectively, resulting in an $\mathrm{EOR}_{\mathrm{obs} 1}, \mathrm{EOR}_{\mathrm{obs} 2}$, and $\mathrm{EOR}_{\mathrm{obs} 3}$. The mean of these three measurements is defined as the $\mathrm{EOR}_{\mathrm{MRI}}$.

\section{Power calculation}

Previous literature comparing the presence of residual tumor on MR images with the estimation made by the neurosurgeon is scarce. We predicted to find an accuracy of $80 \%$, meaning that the estimation about the presence of residual tumor mass should agree with the findings on MRI in $80 \%$ of the cases. Sample size calculation with a $95 \%$ confidence probability, resulted in a sample size of 62 .

\section{Analysis}

All analyses were performed on SPSS software version 25 (IBM Inc., Armonk, New York) for Windows (Microsoft Inc., Redmond, Washington). We have used an altered Bland-Altmann plot to assess agreement between more than two observers [8].

Interobserver agreement for pre-opTV, post-opTV, and EOR was measured using intraclass correlation coefficient (ICC) based on a two-way random model for absolute agreement. Using semi-automated volumetric analysis methods, even the smallest remnants are detected. Therefore, a cut-off value of $99 \%$ was used as a surrogate for residual tumor only for accuracy calculation.

A univariate multivariable general linear model was used to

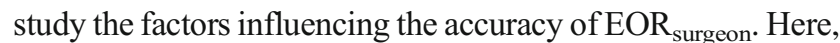
we used $\Delta=\mathrm{EOR}_{\text {surgeon }}-\mathrm{EOR}_{\mathrm{MRI}}$ as the dependent variable. The following variables were included in analysis: Pre-opTV (mean Pre-opTV of the three observers), blood loss during surgery, use of fluorescence, operating neurosurgeon, and $\mathrm{EOR}_{\mathrm{MRI}}$. Significance for all analysis was set at a $p$ value $<$ 0.05 . 


\section{Results}

\section{Participants}

Between November 2012 and May 2018, 62 patients were included in the study. A flowchart of the selection process can be found in Fig. 1. The characteristics of the study group are summarized in Table 1. The group consisted of 38 male $(61 \%)$ and 24 female (39\%) patients. The median age at diagnosis was 63.5 year (range $27-78$ years).

\section{Agreement between EOR obs1 $_{1}, E_{\text {obs2 }}$ and EOR obs3}

The altered Bland-Altmann plot for pre-opTV and post-opTV showed good agreement between observers (see Supplemental Digital Content). An ICC of 0.980 (95\% CI 0.969-0.987) for pre-opTV and 0.974 (95\% CI 0.961-0.984) for post-opTV showed an excellent reliability between observers for these measurements [9].

The Bland-Altmann plot for EOR can be found in Fig. 2. The estimated limits of agreement were -12.39 to 12.39 . The plot shows no systematic over or underestimation of EOR by any observer. There was an excellent reliability for EOR measurement, with an ICC of 0.947 (0.917-0.967) (Table 2).

\section{EOR $_{\text {surgeon, }}$ EOR $_{\text {MRI }}$}

There is a higher agreement between $\mathrm{EOR}_{\text {surgeon }}$ and $\mathrm{EOR}_{\mathrm{MRI}}$ with increasing resection percentage and when fluorescence is used (Fig. 3). The cases are equally distributed above and below the identity line, so there was no systematic EOR over or underestimation by the surgeon.

Presence of residual tumor (defined as EOR $<99 \%$ ) was estimated by the neurosurgeon almost as often as it was seen

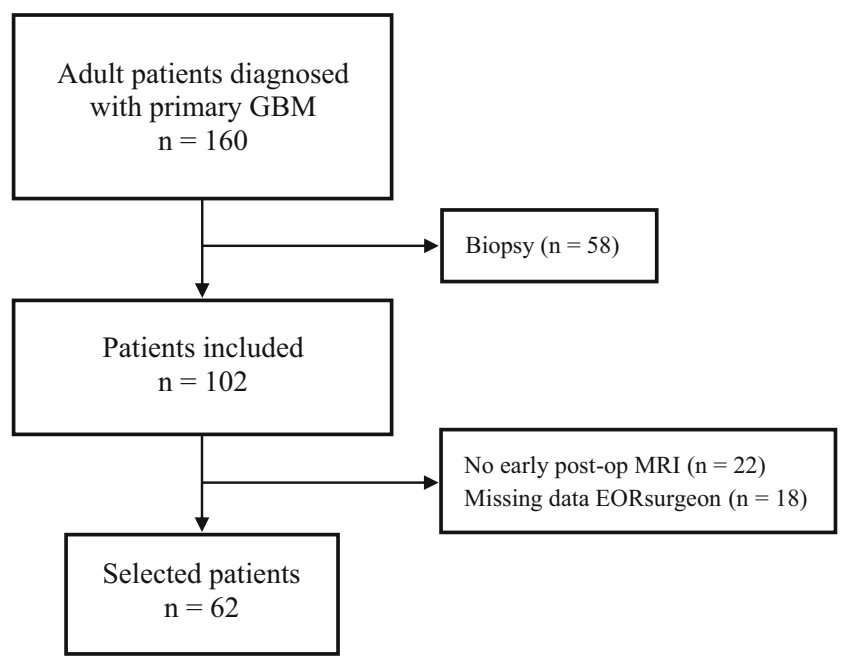

Fig. 1 Flowchart describing the selection process of the participants between November 2012 and May 2018. GBM, glioblastoma. Neurosurgeon, extent of resection estimated by the operating surgeon
Table 1 Patient characteristics $(n=62)$

\begin{tabular}{lcl}
\hline Variable & No. of patients $(\%)$ & \\
\hline Sex & & \\
Male & $38(61)$ & \\
Female & $24(39)$ & 63.5 \\
Age at diagnosis & & $27-78$ \\
Median & & \\
Range & & \\
Side of tumor & $32(52)$ & \\
Left & $30(48)$ & \\
Right & & \\
Tumor location & $18(29)$ & \\
Frontal lobe & $28(45)$ & \\
Temporal lobe & $13(21)$ & \\
Parietal lobe & $2(3)$ & \\
Occipital & $1(2)$ & \\
Basal ganglia & & \\
Necrosis & & \\
No & & \\
Yes & & \\
Fluorescence guided resection (use of 5-ALA) & \\
No & $40(65)$ & \\
Yes & $22(35)$ & \\
Use of neuronavigation & & \\
No & & \\
Yes & & \\
Blood loss (mL) & & \\
Median & & \\
Range & & \\
Pre-operative tumor volume* & & \\
Median & & \\
Range & & \\
\hline
\end{tabular}

Numbers are absolute values (percentages)

*The mean pre-operative volume of neurosurgeon, radiologist, and medical student

on the MRI: surgeons expected residual tumor presence in $76 \%$ of the patients, whilst residual was seen in $79 \%$ of the MR images (Table 3). The accuracy of predicting presence of residual tumor (defined as EOR $<99 \%$ ) was $77 \%$, meaning that $77 \%$ of the estimations about presence of residual tumor was correct.

The ICC for EOR $\mathrm{E}_{\text {surgeon }}$ and $\mathrm{EOR}_{\mathrm{MRI}}$ was $0.641(95 \% \mathrm{CI}$ $0.404-0.784)$. The latter indicates a moderate reliability of the $\mathrm{EOR}_{\text {surgeon }}[9]$.

Multivariable analysis showed a statistically significant effect of operating neurosurgeon $(p=0.01)$, use of fluorescence $(p<0.001)$, and $\mathrm{EOR}_{\mathrm{MRI}}(\mathrm{p}<0.001)$ on $\Delta_{\text {surgeon - MRI. Blood }}$ loss and pre-operative tumor volume were not statically significant factors. 


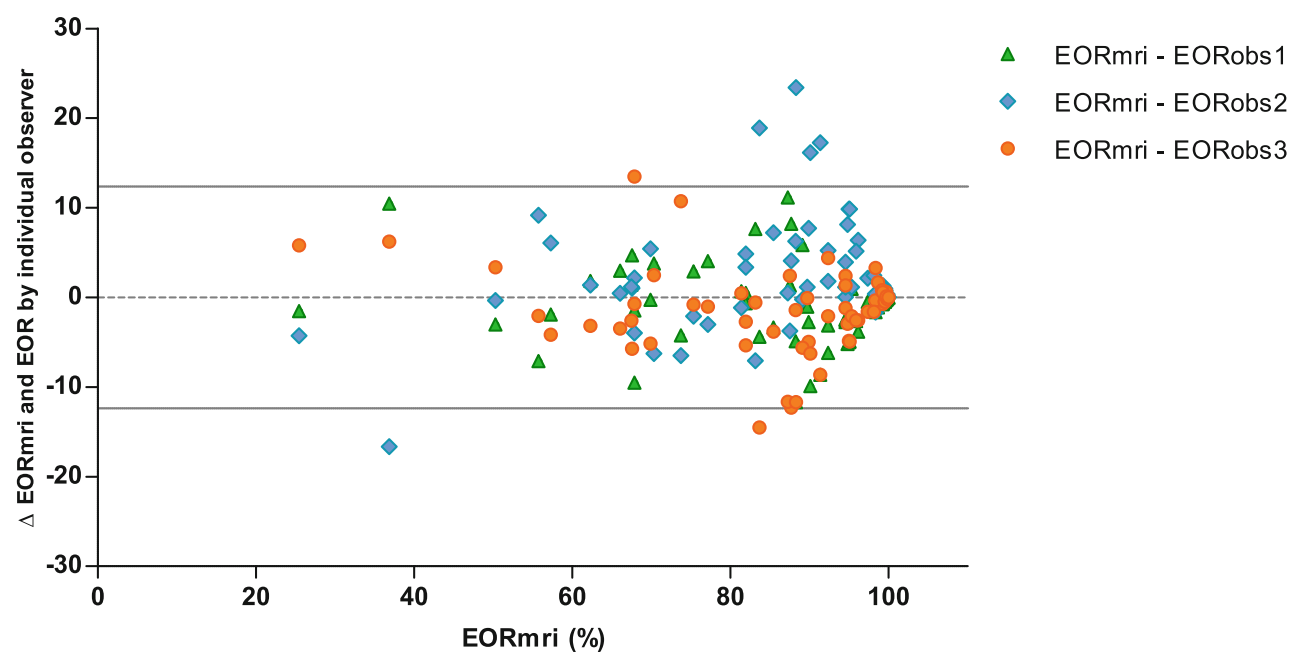

Fig. 2 Bland-Altman plot for three observers [8]. EORmri ( $x$-axis) is plotted against EORmri minus the EOR measured by the individual observer ( $y$-axis). This graph displays per patient the agreement between the three observers. High agreement corresponds with the three colors being close together. The lines represent the limits of agreement. EOR, extent of resection; EORmri, mean EOR of three observers; EORobs1, EOR measured by neurosurgeon; EORobs2, EOR measured by radiologist; EORobs3, EOR measured by student
Following these findings, an estimated marginal means was calculated for use of fluorescence. Use of fluorescence showed an estimated marginal mean of 12.48 (95\% CI 7.26; 17.71), meaning with the use of fluorescence, there is a significant overestimation by the surgeon.

\section{Discussion}

Our main purpose in this study was to compare the EOR estimated by the neurosurgeon post-operatively $\left(\mathrm{EOR}_{\text {surgeon }}\right)$ with the EOR determined using volumetric analysis on the post-operative MR scan (EOR $\left.\mathrm{ERI}_{\text {MI }}\right)$.

In order to achieve this, we first studied the reliability of semi-automated volumetric analysis for determining EOR. There was a very high interobserver agreement between the three observers for measurements of pre-operative tumor volume, post-operative tumor volume, and extent of resection. Therefore, semi-automatic volumetric measurement of extent resection of glioblastoma is reliable.

Our data shows that residual tumor (defined as EOR $<$ 99\%) is seen on MR images as often as suggested by the neurosurgeon. This simple comparison shows an evident result and this way of reporting data is similar to that of the previous study on this subject [7]. However, with the increased precision of statistical analysis in this era, we added a more statistical approach to display our findings. So, we found an accuracy of $77 \%$ and a moderate reliability [9].

Higher resection percentage contributed to a higher agreement between $\mathrm{EOR}_{\text {surgeon }}$ and $\mathrm{EOR}_{\mathrm{MRI}}$. The use of fluorescence also contributed to a higher agreement, with a tendency of overestimation of the EOR by the surgeon (marginal means $12.4895 \%$ CI 7.26 ; 17.71). The reliability of the surgeons' estimate was surgeon dependant.

Using manual segmentation, others have reported high interobserver agreement only for pre-operative tumor volume, but not regarding post-operative volume and EOR [6]. Prior studies using semi-automated methods found a high interobserver agreement for calculation of resection percentage $[4,5]$. Our results agree, showing that semi-automatic volume assessment in GBM is a reliable method for detecting postoperative residual and determining EOR.

Only one prior study has reported on the reliability of surgeons' estimate of EOR [7]. In this study, data collection was nominal: presence of residual tumor was answered with "yes," "no," or "?." Residual tumor was detected 3 times more often
Table 2 Measurements of observers and corresponding intraclass correlation coefficients

\begin{tabular}{lcccc}
\hline & Neurosurgeon & Radiologist & Student & ICC (95\% CI) \\
\hline Mean pre-opTV (SD) & $38.15(33.47)$ & $37.72(29.43)$ & $39.92(33.08)$ & $0.980(0.969-0.987)$ \\
Mean post-opTV (SD) & $6.01(12.01)$ & $5.69(8.25)$ & $5.68(10.71)$ & $0.974(0.961-0.984)$ \\
Mean EOR (SD) & $86.41(17.66)$ & $83.21(16.06)$ & $86.96(17.96)$ & $0.947(0.917-0.967)$ \\
\hline
\end{tabular}

The ICC was calculated using a two-way random model for absolute agreement. Pre-opTV, post-opTV, and EOR all showed a high ICC, indicating an excellent reliability between observers. ICC, intraclass correlation coefficient; Pre-opTV, pre-operative tumor volume; Post-opTV, post-operative tumor volume; EOR, extent of resection 
Fig. 3 Scatterplot for extent of resection (EOR) based on semiautomatic calculation on MR (EORmri) versus EOR estimated by the operating surgeon (EORsurgeon). Triangles represent resections where fluorescence was used, circles represent resections without fluorescence. The identity line $(y=x)$ is shown for reference

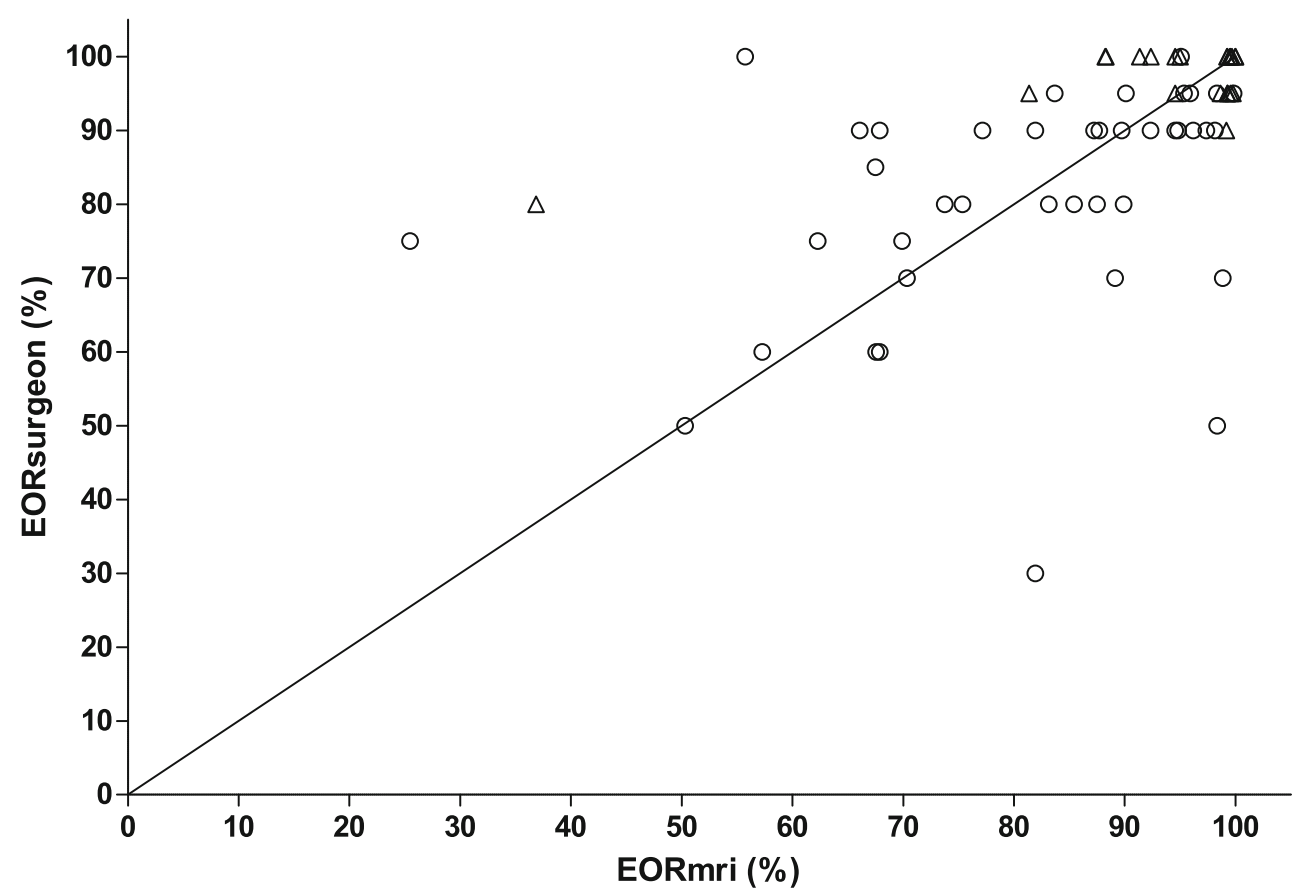

on MRI than estimated by the surgeon. Our data shows the findings of residual tumor on MRI to be equal to the estimation of the neurosurgeon. At first hand, this seems as an improvement in reliability of surgeons' estimate as hypothesized, but further analysis shows only moderate reliability.

The strength of our study is the use of semi-automatic volumetric assessment of EOR and showing this method of determining EOR is highly reliable. Another strength is the statistical

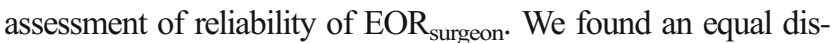
tribution of over and underestimation of EOR by the surgeon, so we did not confirm the systematic overestimation of the surgeon as suggested by others [7]. We did find that accuracy of the surgeons' estimate is surgeon dependant. So, some surgeons estimate the resection percentage better than others. Even though the use of fluorescence resulted in a slight overestimation, it did increase the accuracy of surgeons' estimate.

There were a few limitations in our study. Our study was powered to detect accuracy of $80 \%$, but the low number of subjects limits the factors that can be included in our multivariate analysis. We have calculated accuracy in an indirect way, using a dichotomy with $99 \%$ EOR as the cut-off value. Nevertheless, the different statistical approaches in this study all show a comparable result: moderate agreement for surgeons' estimate and volumetric assessment by MRI. Finally, since residual volume is

Table 3 Assessment of completeness of tumor resection: comparison between the neurosurgeon's estimation and the findings on MRI

\begin{tabular}{lll}
\hline Residual tumor & Neurosurgeon $(\%)$ & MRI $(\%)$ \\
\hline Yes $($ EOR $<99 \%)$ & 76 & 79 \\
No $($ EOR $\geq 99 \%)$ & 24 & 21 \\
\hline
\end{tabular}

clinically more relevant than percentages, we have plotted surgeons estimating complete resection against residual volume. There was no estimation of complete resection when residual was above $6 \mathrm{~mL}$. With minimal residual volume $(<2 \mathrm{~mL}, 2-$ $4 \mathrm{~mL}$, and $4-6 \mathrm{~mL}$ ), 17 to $36 \%$ of surgeons estimated a complete resection (Supplemental Digital Content, Fig. 3).

\section{Conclusion}

Semi-automatic volumetric analysis showed a high interobserver agreement and should therefore be considered the gold standard for assessment of EOR. The introduction of fluorescence has resulted in better resections [10]. We found it to increase the accuracy of the surgeons' estimate of fluorescence, whilst resulting in a tendency towards overestimation. Even though surgeons' estimate of extent of resection has clearly improved since the report of Albert et al., the reliability of their estimation is statistically moderate. Therefore, early post-operative MRI scanning for evaluation of EOR remains paramount.

\section{Compliance with ethical standards}

Conflict of interest The authors declare that they have no conflict of interest.

Ethical approval All procedures performed in studies involving human participants were in accordance with the ethical standards of the institutional and/or national research committee and with the 1964 Helsinki declaration and its later amendments or comparable ethical standards.

Informed consent For this type of study, formal consent is not required. 
Open Access This article is distributed under the terms of the Creative Commons Attribution 4.0 International License (http:// creativecommons.org/licenses/by/4.0/), which permits unrestricted use, distribution, and reproduction in any medium, provided you give appropriate credit to the original author(s) and the source, provide a link to the Creative Commons license, and indicate if changes were made.

\section{References}

1. Ostrom QT, Gittleman H, Liao P, Vecchione-Koval T, Wolinsky Y, Kruchko C, Barnholtz-Sloan JS (2017) CBTRUS statistical report: primary brain and other central nervous system tumors diagnosed in the United States in 2010-2014. Neuro-Oncology 19:v1-v88. https://doi.org/10.1093/neuonc/nox158

2. Sanai N, Polley MY, McDermott MW, Parsa AT, Berger MS (2011) An extent of resection threshold for newly diagnosed glioblastomas. J Neurosurg 115:3-8. https://doi.org/10.3171/2011.2. jns1099810.3171/2011.7.jns10238

3. Stummer W, van den Bent MJ, Westphal M (2011) Cytoreductive surgery of glioblastoma as the key to successful adjuvant therapies: new arguments in an old discussion. Acta Neurochir 153:12111218. https://doi.org/10.1007/s00701-011-1001-x

4. Chow DS, Qi J, Guo X, Miloushev VZ, Iwamoto FM, Bruce JN, Lassman AB, Schwartz LH, Lignelli A, Zhao B, Filippi CG (2014) Semiautomated volumetric measurement on postcontrast MR imaging for analysis of recurrent and residual disease in glioblastoma multiforme. AJNR Am J Neuroradiol 35:498-503. https://doi.org/ 10.3174/ajnr.A3724
5. Kanaly CW, Mehta AI, Ding D, Hoang JK, Kranz PG, Herndon JE 2nd, Coan A, Crocker I, Waller AF, Friedman AH, Reardon DA, Sampson JH (2014) A novel, reproducible, and objective method for volumetric magnetic resonance imaging assessment of enhancing glioblastoma. J Neurosurg 121:536-542. https://doi.org/10. 3171/2014.4.Jns121952

6. Kubben PL, Postma AA, Kessels AG, van Overbeeke JJ, van Santbrink H (2010) Intraobserver and interobserver agreement in volumetric assessment of glioblastoma multiforme resection. Neurosurgery 67:1329-1334. https://doi.org/10.1227/NEU. 0b013e3181efbb08

7. Albert FK, Forsting M, Sartor K, Adams HP, Kunze S (1994) Early postoperative magnetic resonance imaging after resection of malignant glioma: objective evaluation of residual tumor and its influence on regrowth and prognosis. Neurosurgery 34:45-60 discussion 6041

8. Jones M, Dobson A, O'Brian S (2011) A graphical method for assessing agreement with the mean between multiple observers using continuous measures. Int J Epidemiol 40:1308-1313. https://doi.org/10.1093/ije/dyr109

9. Koo TK, Li MY (2016) A guideline of selecting and reporting intraclass correlation coefficients for reliability research. J Chiropr Med 15:155-163. https://doi.org/10.1016/j.jcm.2016.02.012

10. Eljamel S (2015) 5-ALA fluorescence image guided resection of glioblastoma multiforme: a meta-analysis of the literature. Int J Mol Sci 16:10443-10456. https://doi.org/10.3390/ijms160510443

Publisher's note Springer Nature remains neutral with regard to jurisdictional claims in published maps and institutional affiliations. 